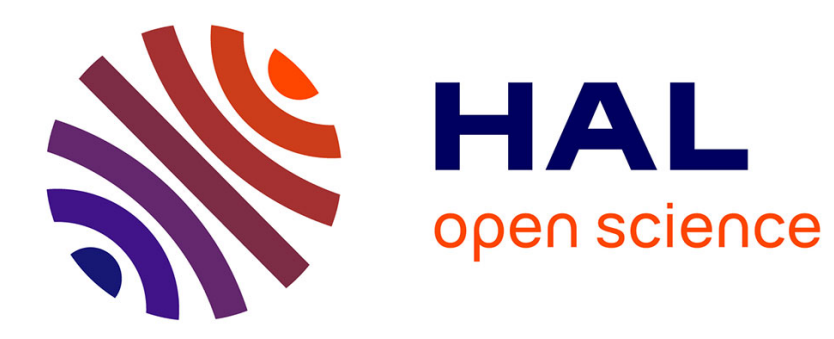

\title{
Genericity of weakly computable objects Mathieu Hoyrup
}

\section{To cite this version:}

Mathieu Hoyrup. Genericity of weakly computable objects. Theory of Computing Systems, 2017, Special Issue: Theoretical Aspects of Computer Science, 60 (3), 10.1007/s00224-016-9737-6 . hal$01095864 \mathrm{v} 2$

\section{HAL Id: hal-01095864 https://hal.inria.fr/hal-01095864v2}

Submitted on 9 Dec 2016

HAL is a multi-disciplinary open access archive for the deposit and dissemination of scientific research documents, whether they are published or not. The documents may come from teaching and research institutions in France or abroad, or from public or private research centers.
L'archive ouverte pluridisciplinaire HAL, est destinée au dépôt et à la diffusion de documents scientifiques de niveau recherche, publiés ou non, émanant des établissements d'enseignement et de recherche français ou étrangers, des laboratoires publics ou privés. 


\title{
Genericity of weakly computable objects
}

\author{
Mathieu Hoyrup
}

October 12, 2016

\begin{abstract}
In computability theory many results state the existence of objects that in many respects lack algorithmic structure but at the same time are effective in some sense. Friedberg and Muchnik's answer to Post's problem is one of the most celebrated results in this form. The main goal of the paper is to develop a general result that embodies a large number of these particular constructions, capturing the essential idea that is common to all of them, and expressing it in topological terms.

To do so, we introduce the effective topological notions of irreversible function and directional genericity and provide two main results that identify situations when such constructions are possible, clarifying the role of topology in many arguments from computability theory. We apply these abstract results to particular situations, illustrating their strength and deriving new results.

This paper is an extended version of the conference paper [Hoy14] with detailed proofs and new results.
\end{abstract}

\section{Contents}

1 Introduction $\quad 2$

2 Background and notations $\quad 3$

2.1 Notations . . . . . . . . . . . . . . . . . . 4

2.2 Effective topology . . . . . . . . . . . . . . . . 4

2.3 Effective Polish spaces . . . . . . . . . . . . . . . 4

$\begin{array}{lll}3 & \text { Irreversible functions } & \mathbf{7}\end{array}$

3.1 A non-uniform result . . . . . . . . . . . . . . 7

3.2 Irreversible functions . . . . . . . . . . . . . . . . 9

3.3 The constructive result . . . . . . . . . . . . . . 11

3.4 Application to the ergodic decomposition . . . . . . . . . . 14 
4 Directional genericity $\quad 16$

4.1 Being generic from above . . . . . . . . . . . . . 16

4.2 Genericity for c.e. sets . . . . . . . . . . . . . . 21

4.3 Genericity for left-c.e reals . . . . . . . . . . . . . 22

4.4 Genericity for $\Pi_{1}^{0}$-classes . . . . . . . . . . . . . . . . . 25

4.5 Genericity for regular $\Pi_{1}^{0}$-classes $\ldots \ldots . \ldots . . \ldots 26$

\section{Introduction}

One of the main goals of computability theory is to understand and classify the algorithmic content of infinite objects, which can be expressed as the difficulty of computing them or as their ability to help solving problems. In establishing this classification one is often led to separate classes of algorithmic complexity and the construction of counter-examples is usually a hard task that requires the use of advanced techniques, one of the simplest being the so-called priority method with finite injury. The difficulty in carrying out such constructions is that the built object should meet two types of requirements going in opposite directions: (i) it should lack algorithmic content but at the same time (ii) it should be constructible in some way. In other words, these objects live somewhere between generic objects (objects with no structure) and computable objects (the most constructible objects). While computability theory provides formal notions of genericity, these ones are always incompatible with computability, so the two prescriptions are conflicting.

In this paper we propose an abstract view on this topic by providing general results that, when applied properly, implement the sought constructions, and provide a shortcut for producing objects with prescribed properties. We expect that checking the conditions of these theorems is much easier than writing down the complete construction which is often merely an adaptation of known techniques to new situations.

The first main result (Theorem 3.3.1) enables one to construct objects that (i) are not computable, but (ii) have a computable image under some given function. As an illustration, we prove a negative result about the computability of the ergodic decomposition. This part is developed in Section 3 and is the version with detailed proofs of the results already presented in [Hoy14].

The second main result (Theorem 4.1.1) enables one to construct objects that (i) are generic in some sense, but (ii) are computable in a weak sense. It is done by introducing a new notion of genericity, called directional generic- 
ity, that has two advantages: (i) it is formally close to plain genericity, being defined as a slight variation of 1 -genericity, and (ii) it is compatible with various weak forms of computability. For instance for subsets of $\mathbb{N}$, directional genericity is called genericity from above and we prove the existence of c.e. sets that are generic from above. Such sets are in particular solutions to Friedberg-Muchnik's theorem. Theorem 4.1.1 has important consequences:

- It subsumes and unifies many ad hoc existing constructions,

- It can be applied to obtain new results,

- It clarifies the role of topology in computability theory.

We then illustrate these notions of genericity in several contexts, showing evidence that they clarify existing results, and we apply our main theorem to derive a separation result. This part is developed in Section 4 and is a significant extension of what appears in [Hoy14].

The proofs of our main results use the priority method with finite injury. We observe that while this technique has been massively used in many situations, no result has convincingly clarified its scope and the contexts in which it can be applied, and each particular construction is yet another adaptation of the same argument, based on the same idea. We hope that the results presented here shed light on this subject.

The paper is organized as follows. In Section 2 we present the needed background on computable analysis, providing along the way a few results of independent interest on effective Polish spaces. In Section 3 we introduce the notion of irreversible function and prove the first main result: if a computable function is effectively irreversible then it maps a non-computable point to a computable image. The results of this section come from [Hoy14], we present here their detailed proofs. In Section 4 we introduce a notion of genericity. We prove the second main result of the paper: under suitable assumptions, a point exists that is at the same time "generic" and "weakly computable". We then apply this result to four classes of objects: c.e. sets, left-c.e. reals, $\Pi_{1}^{0}$-classes and regular $\Pi_{1}^{0}$-classes.

\section{Background and notations}

We assume familiarity with basic computability theory on the natural numbers. We implicitly use Weihrauch's notions of computability on effective topological spaces, based on the standard representation (see [Wei00] for more details), however we do not express them in terms of representations. 


\section{$2.1 \quad$ Notations}

In a metric space $(X, d)$, if $x \in X$ and $r \in(0,+\infty)$ then we denote the open ball with center $x$ and radius $r$ by $B(x, r)=\left\{x^{\prime} \in X: d\left(x, x^{\prime}\right)<r\right\}$. We denote the corresponding closed ball by $\bar{B}(x, r)=\left\{x^{\prime} \in X: d\left(x, x^{\prime}\right) \leq r\right\}$. The Cantor space of infinite binary sequences, or equivalently subsets of $\mathbb{N}$, is denoted by $2^{\mathbb{N}}$. The halting set, denoted $\emptyset^{\prime}$, is the set of numbers of Turing machines that halt upon input of their own number. It is a noncomputable set that is computably enumerable (c.e.).

\section{$2.2 \quad$ Effective topology}

An effective topological space $(X, \tau, \mathcal{B})$ consists of a topological space ${ }^{1}(X, \tau)$ together with a countable basis $\mathcal{B}=\left\{B_{0}, B_{1}, \ldots\right\}$ numbered in such a way that the finite intersection operator is computable: for each $i, j$ there exists a set $W \subseteq \mathbb{N}$ that is c.e. uniformly in $(i, j)$, such that $B_{i} \cap B_{j}=\bigcup_{k \in W} B_{k}$. An open subset $U \subseteq X$ is effectively open if $U=\bigcup_{k \in W} B_{k}$ for some c.e. set $W \subseteq \mathbb{N}$.

To a point $x \in X$ we associate $N(x)=\left\{n \in \mathbb{N}: x \in B_{n}\right\}$. By an enumeration of $N(x)$ we mean a total function $f: \mathbb{N} \rightarrow \mathbb{N}$ whose range is $N(x)$. A point $x$ is computable if $N(x)$ is c.e., i.e. if $N(x)$ has a computable enumeration.

Given points $x, y$ in effective topological spaces $X, Y$ respectively, we say that $y$ is computable relative to $x$ if there is an oracle Turing machine $M$ that, given any enumeration of $N(x)$ as an oracle, outputs an enumeration of $N(y)$. We denote it by $M^{x}=y$. In other words, $y$ is computable relative to $x$ if $N(y)$ is enumeration reducible to $N(x)$. As proved by Selman [Sel71] and pointed out by Miller [Mil04], $y$ is computable relative to $x$ if and only if every enumeration of $N(x)$ computes an enumeration of $N(y)$ (uniformity is not explicitly required, but is a consequence).

A (possibly partial) function $f: X \rightarrow Y$ is computable if there is a machine $M$ such that for every $x \in \operatorname{dom}(f), M^{x}=f(x)$. A computable function is always continuous.

\subsection{Effective Polish spaces}

An effective Polish space is a topological space such that there exists a dense sequence $s_{0}, s_{1}, \ldots$ of points, called simple points and a complete metric $d$ inducing the topology, such that all the reals numbers $d\left(s_{i}, s_{j}\right)$ are

\footnotetext{
${ }^{1}$ Such spaces are usually assumed to be $T_{0}$, however this assumption is not necessary.
} 
computable uniformly in $(i, j)$. Every effective Polish space can be made an effective topological space, taking as canonical basis the open balls $B(s, r)$ with $s$ as a simple point and with $r$ as a positive rational number together with a standard effective numbering. It is sometimes useful to use another basis: we say that a family $\mathcal{B}=\left\{B_{i}\right\}_{i \in \mathbb{N}}$ of open sets is an effective basis if the identity between the effective topological spaces induced by $\mathcal{B}$ and by the canonical basis is computable in the two directions. For instance,

Proposition 2.3.1. There always exists an effective basis $\mathcal{B}$ that makes the predicate $s_{i} \in B_{j}$ computable in $i, j$.

Proof. By a standard diagonalization there exists a computable dense sequence of positive real numbers $r_{n}$ such that $d\left(s_{i}, s_{j}\right) \neq r_{n}$ for all $n$. Take the basis of metric balls centered at simple points with radii in the sequence $r_{n}$.

In an effective Polish space, a point $x$ is computable if and only if for every $\epsilon>0$ a simple point $s$ can be computed, uniformly in $\epsilon$, such that $d(s, x)<\epsilon$.

We present a few simple observations on effective Polish spaces which seem to be new and are of independent interest. Let $X$ be an effective Polish space. $X^{\prime} \subseteq X$ is an effective Polish subspace if it is an effective Polish space under the induced topology and such that the canonical injection from $X^{\prime}$ to $X$ is a computable homeomorphism between $X^{\prime}$ as a space and $X^{\prime}$ as a subset of $X$. Alexandrov's theorem gives a way to obtain Polish subspaces of a Polish space, and has an effective version, which we present now.

A set $A$ is an effective $G_{\delta}$-set if there exists a family of uniformly effective open sets $U_{n}$ such that $A=\bigcap_{n} U_{n}$. The following result unifies two known results: every closed set whose hit set (see statement below) is c.e. contains a dense computable sequence; every dense effective $G_{\delta \text {-set }}$ contains a dense computable sequence (computable Baire Category theorem [YMT99, Bra01]).

Proposition 2.3.2. Let $A$ be an effective $G_{\delta}$-subset of an effective Polish space. The hit set $\left\{i \in \mathbb{N}: B_{i} \cap A \neq \emptyset\right\}$ is c.e. if and only if $A$ contains a computable sequence dense in $A$. We then say that $A$ is c.e.

Proof. We prove the non-trivial direction. Let $U_{n}$ be uniformly effective open sets and $A=\bigcap_{n} U_{n}$, such that $\left\{i \in \mathbb{N}: B_{i} \cap A \neq \emptyset\right\}$ is c.e. Given a ball $B_{0}$ intersecting $A$, one can compute a sequence of balls $B_{n}$ of radius $<2^{-n}$ such that $\bar{B}_{n+1} \subseteq B_{n} \subseteq U_{n}\left(\bar{B}_{n+1}\right.$ is the closed ball rather 
than the closure of $\left.B_{n+1}\right)$. The intersection $\bigcap_{n} B_{n}$ contains one point that is computable and belongs to $A$. The induction hypothesis on $B_{n}$ is that it intersects $A$. Once $B_{n}$ has been built, there must be some ball $B$ of radius $<2^{-n-1}$ such that $\bar{B} \subseteq B_{n} \cap U_{n}$ and $B$ intersects $A$, and such a $B$ can be effectively computed. Let $B_{n+1}=B$. To obtain a dense computable sequence, start from any ball $B_{0}$ intersecting $A$.

We will often use the same technic to build a point, by means of a shrinking sequence of balls. A sequence $B_{n}$ of balls is shrinking if the radius of $B_{n}$ converges to 0 and $\bar{B}_{n+1} \subseteq B_{n}$. By completeness of the metric, $\bigcap_{n} B_{n}$ always contains a point.

Proposition 2.3.3 (Effective Alexandrov Theorem). Let $X$ be an effective Polish space. Every c.e. effective $G_{\delta}$-set is an effective Polish subspace of $X$.

Proof. The proof is a simple effective version of the proof that can be found in [HY61] (Theorem 2-76 therein). Let $A=\bigcap_{n} U_{n}$ be a c.e. effective $G_{\delta}$-set. Let $d$ be a complete computable metric on $X$. Let $d_{n}: X \rightarrow[0,+\infty)$ be uniformly computable functions such that $d_{n}(x)>0 \Longleftrightarrow x \in U_{n}$. One can think of $d_{n}$ as a computable version of the distance to the complement of $U_{n}$. If $U_{n}=\bigcup_{i} B\left(x_{i}, r_{i}\right)$ then one can define $d_{n}$ as $d_{n}(x)=\sum_{i} 2^{-i} \max (1-$ $\left.d\left(x, x_{i}\right) / r_{i}, 0\right)$. For $x \in U_{n}$, let $f_{n}(x)=\frac{1}{d_{n}(x)}$. For $x, y \in A$, let

$$
d^{\prime}(x, y)=d(x, y)+\sum_{n} 2^{-n} \frac{\left|f_{n}(x)-f_{n}(y)\right|}{1+\left|f_{n}(x)-f_{n}(y)\right|} .
$$

The function $d^{\prime}: A \times A \rightarrow \mathbb{R}$ is computable. On $A$ it is a complete metric that induces the same topology as $d$. The computable sequence which is dense in $A$ can serve as special points in $A$. The canonical injection is 1-Lipschitz, hence has a computable modulus of uniform continuity and is computable on the special points of $A$, so it is computable.

The statement in the theorem can actually be extended to an equivalence.

We will be concerned with computability and Baire category, so we will naturally meet the notion of a 1-generic point: a point that does not belong to any "effectively meager set" in the following sense.

Definition 2.1. $x \in X$ is 1-generic if $x$ does not belong to the boundary of any effective open set. In other words, for every effective open set $U$, either $x \in U$ or there exists a neighborhood $B$ of $x$ disjoint from $U$. 
By the Baire category theorem, every Polish space is a Baire space so 1generic points exist and form a co-meager set. One can relativize this notion by considering all the open sets that are effective relative to a given oracle.

A weaker notion of genericity is useful.

Definition 2.2. $x \in X$ is weakly 1-generic if $x$ belongs to every dense effective open set.

\section{Irreversible functions}

\subsection{A non-uniform result}

Let $X$ be an effective Polish space, $Y$ an effective topological space and $f$ : $X \rightarrow Y$ a (total) computable function.

To introduce the results of this section informally, assume temporarily that $f$ is one-to-one. If $f^{-1}$ is computable, i.e. if every $x$ is computable relative to $f(x)$ uniformly in $f(x)$, then $f^{-1}$ is continuous. As mentioned earlier uniformity is crucial here: that some $x$ is computable relative to $f(x)$ does not imply in general that $f^{-1}$ is continuous at $f(x)$. Theorem 3.1.1 below surprisingly shows that a non-uniform version can still be obtained, valid at most points.

Let us now make it precise and formal. We do not assume anymore that $f$ is one-to-one.

When focusing on the problem of inverting a function, one comes naturally to the following basic notions:

- $f$ is invertible at $x$ if $x$ is the only pre-image of $f(x)$,

- $f$ is locally invertible at $x$ if $x$ is isolated in the pre-image of $f(x)$.

If one has access to $x$ via its image only, then $x$ is determined unambiguously in the first case, with the help of a discrete advice (a basic open set isolating $x$ ) in the second case. However, "being uniquely determined" is not sufficient in practice: physically or computationally, one cannot entirely know $f(x)$ in one step, but progressively as a limit of finite approximations. We need to consider stronger, topological versions of the two basic notions of invertibility, expressing that $x$ can be recovered from the knowledge of its image given by finer and finer neighborhoods.

Definition 3.1. Let $f: X \rightarrow Y$ be a function.

We say that $f$ is continuously invertible at $x$ if the pre-images of the neighborhoods of $f(x)$ form a neighborhood basis of $x$, i.e. for every neighborhood $U$ of $x$ there exists a neighborhood $V$ of $f(x)$ such that $f^{-1}(V) \subseteq U$. 
We say that $f$ is locally continuously invertible at $x$ if there exists a neighborhood $B$ of $x$ such that the restriction of $f$ to $B$ is continuously invertible at $x$, i.e. for every neighborhood $U$ of $x$ there exists a neighborhood $V$ of $f(x)$ such that $B \cap f^{-1}(V) \subseteq U$.

Observe that these notions are very natural when investigating the problem of inverting a function: we think that they are not technical ad hoc conditions.

Every effective topological space $Y$ has a countable basis hence is sequential, i.e. continuity notions can be expressed in terms of sequences, which may be more intuitive. We will be particularly interested in the negations of these notions, which we characterize now, using any metric $d$ generating the topology.

Proposition 3.1.1. $f$ is not continuously invertible at $x$ if and only if there exists $\delta>0$ and a sequence $x_{n}$ such that $d\left(x, x_{n}\right)>\delta$ and $f\left(x_{n}\right)$ converges to $f(x)$.

$f$ is not locally continuously invertible at $x$ if and only if for every $\epsilon>0$ there exists $\delta>0$ and a sequence $x_{n}$ such that $\epsilon>d\left(x, x_{n}\right)>\delta$ and $f\left(x_{n}\right)$ converges to $f(x)$.

We now come to our first result.

Theorem 3.1.1 (Computability implies continuity, pointwise). Let $f: X \rightarrow$ $Y$ be a computable function and $x \in X$ a 1-generic point.

If $x$ is computable relative to $f(x)$ then $f$ is locally continuously invertible at $x$.

Proof. Assume that $x$ is computable relative to $f(x)$ and $f$ is not locally continuously invertible at $x$. We show that $x$ belongs to the boundary of an effective open set $U$, i.e. that $x$ is not 1-generic.

Intuitively, for a point $y$, there are two possible ways in which a Turing machine $M$ may fail to compute $y$ from $f(y)$ : either it diverges, or it outputs something that is incompatible with $y$. The latter can be recognized in finite time: we then say that $M^{f(y)}$ positively fails to compute $y$. Our effective open set $U$ will be the set of points $y$ such that $M^{f(y)}$ positively fails to compute $y$.

Let us make it more precise. As $x$ is computable relative to $f(x)$, there exist uniformly effective open sets $V_{n} \subseteq Y$ such that for all $n, x \in B_{n} \Longleftrightarrow$ $f(x) \in V_{n}$, where $B_{n}$ is the canonical basis induced by a complete effective metric on $X$. We then define

$$
U=\bigcup_{n} f^{-1}\left(V_{n}\right) \backslash \bar{B}_{n}
$$


which is an effective open set (if $B_{n}=B(x, r)$ then $\bar{B}_{n}=\bar{B}(x, r)$ is the corresponding closed ball). By definition of $V_{n}, x$ does not belong to $U$.

Let us show that $x$ belongs to the closure of $U$. Let $B$ be a neighborhood of $x$ and $U_{B}$ another neighborhood coming from the fact that $f$ is not locally continuously invertible at $x$. Let $B_{n}$ be a neighborhood of $x$ such that $\bar{B}_{n} \subseteq$ $U_{B}$. The set $V_{n}$ is a neighborhood of $f(x)$, so $f^{-1}\left(V_{n}\right)$ intersects $B \backslash U_{B} \subseteq$ $B \backslash \bar{B}_{n}$. As a result, $B$ intersects $U$. This is true for every neighborhood $B$ of $x$, so $x$ belongs to the closure of $U$.

In the sequel we introduce a condition on $f$ which roughly means that $f$ is "almost nowhere" locally continuously invertible and that entails (i) the existence of an $x$ that is not computable relative to $f(x)$ (Theorem 3.2.1) and, better, (ii) the existence of a non-computable $x$ such that $f(x)$ is computable (Theorem 3.3.1).

\subsection{Irreversible functions}

We now consider the following notion: an irreversible function is locally continuously invertible at almost no point, in the sense of Baire category.

Definition 3.2. $f: X \rightarrow Y$ is irreversible if for every non-empty open set $B \subseteq X$ there exists a non-empty open set $U_{B} \subseteq B$ such that there is no open set $V \subset Y$ satisfying $\emptyset \neq f^{-1}(V) \cap B \subseteq U_{B}$.

In other words, if the pre-image of an open set intersects $B$ then it intersects $B \backslash U_{B}$.

Intuitively, in a game between a player progressively describing $f(x)$ for some $x \in U_{B}$ and an opponent trying to progressively guess $x$, the opponent can never guess that $x \in U_{B}$ even knowing that $x \in B$.

An application of an irreversible function $f$ to $x$ comes with a loss of information about $x$, that can hardly be recovered. Being irreversible is orthogonal to not being one-to-one: the function $x \mapsto x^{2}$ is not one-toone but not irreversible: $x$ can be (continuously or computably) recovered from $x^{2}$; a one-to-one function can be irreversible if its inverse is dramatically discontinuous (examples of such functions will be encountered in the sequel).

In terms of sequences, $f$ is irreversible if and only if for every $B$ there exists a non-empty open set $U_{B} \subseteq B$ such that for every $x \in U_{B}$ there is a sequence $x_{n} \in B \backslash U_{B}$ such that $f\left(x_{n}\right)$ converges to $f(x)$.

As announced, the set of points at which an irreversible function is locally continuously invertible is small in the sense of Baire category. 
Proposition 3.2.1. Let $f$ be irreversible. There is a dense $G_{\delta}$-set $D$ such that $f$ is not locally continuously invertible at any $x \in D$.

Proof. Let $W_{n}$ be the union of $U_{B}$ for all basic open sets $B$ of radius < $2^{-n}$. $W_{n}$ is a dense open set. Let $x \in D:=\bigcap_{n} W_{n}$. For each $n$ there is a ball $B$ of radius $<2^{-n}$ such that $x \in U_{B}$. For every neighborhood $V$ of $f(x), x \in f^{-1}(V) \cap B \neq \emptyset$ so $f^{-1}(V) \cap B \nsubseteq U_{B}$.

In other words, for almost every $x$ the application of $f$ to $x$ comes with a "topological information" loss.

The preceding proposition does not rule out the possibility that the restriction of $f$ to a "large" set have a continuous inverse (for instance, the characteristic function of the rational numbers is nowhere continuous, but its restriction to the co-meager set of irrational numbers is continuous). The next assertion shows that this is not possible.

Proposition 3.2.2. Let $f$ be irreversible and $C \subseteq X$ be such that $f_{\mid C}: C \rightarrow$ $f(C)$ is a homeomorphism. Then $C$ is nowhere dense.

Proof. Assume that the closure of $C$ contains a ball $B$. Let $x \in U_{B} \cap C$. There exists a sequence $x_{n} \in B \backslash U_{B}$ such that $f\left(x_{n}\right)$ converges to $f(x)$. By density of $C$ in $B, x_{n}$ can be taken in $C$. As $f_{\mid C}$ is a homeomorphism and $f\left(x_{n}\right)$ converges to $f(x), x_{n}$ should converge to $x$ and eventually enter $U_{B}$, which gives a contradiction.

In the definition of an irreversible function (Definition 3.2), $B$ and $U_{B}$ can be assumed w.l.o.g. to be basic balls, in some fixed effective basis. We can make Definition 3.2 effective by requiring that given an index for $B$ in the numbered basis, one can compute an index for $U_{B}$.

Definition 3.3. $f$ is effectively irreversible if an index for $U_{B}$ can be computed from an index for $B$ in Definition 3.2.

Proposition 3.2.3. Being effectively irreversible does not depend on the effective basis.

Proof. Let $\mathcal{B}$ and $\mathcal{B}^{\prime}$ be effective bases, and assume that $f$ is effectively irreversible w.r.t. $\mathcal{B}$. Let us show that $f$ is effectively irreversible w.r.t. $\mathcal{B}^{\prime}$. Given $i$, we want to compute $j$ such that $B_{j}^{\prime}$ plays the role of $U_{B_{i}^{\prime}}$, i.e. $B_{j}^{\prime} \subseteq B_{i}^{\prime}$ and no open set $V \subseteq Y$ satisfies $\emptyset \neq f^{-1}(V) \cap B_{i}^{\prime} \subseteq B_{j}^{\prime}$. First, $B_{i}^{\prime}$ is effectively open in the basis $\mathcal{B}$ so one can find $k$ such that $B_{k} \subseteq B_{i}^{\prime}$. As $f$ is effectively irreversible w.r.t. $\mathcal{B}$ one can compute $l$ such that $B_{l}$ plays the role of $U_{B_{k}}$. As $B_{l}$ is effectively open in the basis $\mathcal{B}^{\prime}$ one can find $j$ such that $B_{j}^{\prime} \subseteq$ 
$B_{l}$. This is the sought $j$. Indeed, if there is an open set $V \subseteq Y$ such that $\emptyset \neq f^{-1}(V) \cap B_{i}^{\prime} \subseteq B_{j}^{\prime}$, then as $B_{j}^{\prime} \subseteq B_{l} \subseteq B_{k}, \emptyset \neq f^{-1}(V) \cap B_{k} \subseteq B_{l}$ contradicting the choice of $B_{l}$.

The following result is the effective version of Proposition 3.2.2.

Theorem 3.2.1. If $f$ is computable and effectively irreversible then for every 1-generic $x, x$ is not computable relative to $f(x)$.

Proof. The dense $G_{\delta}$-set provided by Proposition 3.2.1 is effective when $f$ is effectively irreversible so it contains every 1-generic point (even every weakly-1-generic point). Hence for every 1-generic $x, f$ is not locally continuously invertible at $x$. We now apply Theorem 3.1.1.

In other words, if $x$ is 1-generic then the application of $f$ to $x$ comes with an "algorithmic information" loss. So if $f$ is effectively irreversible then there exists some $x$ that is not computable relative to $f(x)$.

\subsection{The constructive result}

We now present the main result of the paper. It is the constructive version of Theorem 3.2.1 as it makes $f(x)$ computable. The construction uses a priority argument with finite injury.

Theorem 3.3.1. If $f$ is computable and effectively irreversible then there exists a non-computable $x$ such that $f(x)$ is computable.

The proof uses the priority method with finite injury, which can be seen as a game between a player, computing $f(x)$, and infinitely many opponents (all the Turing machines) trying to compute $x$. The remainder of this section is devoted to the detailed proof of Theorem 3.3.1.

Here we take as effective basis the balls induced by a complete effective metric of $X$, so that every shrinking sequence of open sets has non-empty intersection. We fix a one-to-one computable enumeration $n_{0}, n_{1}, \ldots$ of the halting set $\emptyset^{\prime}$. We construct $x \in X$ such that $f(x)$ is computable and $\emptyset^{\prime}$ is computable relative to $x$. We construct a shrinking sequence of metric balls $B_{n}$ and define $x$ as the unique member of their intersection. Of course, the sequence $B_{n}$ must not be computable otherwise $x$ would be computable. The sequence $B_{n}$ is constructed in stages: at stage $s$ we define $B_{n}[s]$ and for each $n$ the sequence $B_{n}[s]$ is eventually constant, with limit $B_{n}$. For each $s$, the sequence $B_{n}[s]$ is shrinking, so the limiting sequence $B_{n}$ will be shrinking as well. One may imagine, for each $s$, the sequence $B_{n}[s]$ as an 
infinite path in a tree. At stage $s+1, n_{s}$ is enumerated into $\emptyset^{\prime}$ and the current path branches at depth $n_{s}$.

In order to make $f(x)$ computable we enumerate along the construction the indices of all its basic neighborhoods into a list $L \subseteq \mathbb{N}$. $L$ is the union of a computable growing sequence of finite lists $L_{s}$. At stage $s$, the current neighborhood of $f(x)$, denoted by $V_{s}$, is the (finite) intersection of the basic open sets indexed by $L_{s}$. As $L_{s} \subseteq L_{s+1}, V_{s+1} \subseteq V_{s}$.

We need to consider two technical points. First we use a particular set of special points, induced by the effective irreversibility of $f$, obtained as follows. We can assume w.l.o.g. that the radius of $U_{B}$ is at most half the radius of $B$, that the closure of $U_{B}$ is contained in $B$ and that there is no open set $V \subseteq Y$ such that $\emptyset \neq f^{-1}(V) \cap B \subseteq \bar{U}_{B}$ (if $U_{B}=B(x, r)$ then replace it by $B(x, r / 2))$. Given a basic ball $B$, consider the computable sequence $U_{B}^{(n)}$ defined inductively by $U_{B}^{(0)}=B$ and $U_{B}^{(n+1)}=U_{U_{B}^{(n)}}$. $U_{B}^{(n)}$ is a computable shrinking sequence and the unique member $a$ of $\bigcap_{n} U_{B}^{(n)}$ is computable, uniformly in $B$. The canonical enumeration $B_{j}$ of basic balls induces a computable dense sequence $a_{j}$, which will serve as simple points.

We then come to the second technical point. Let $\left(B_{k}^{\prime}\right)_{k \in \mathbb{N}}$ be the canonical enumeration of the basic open subsets of $Y$. We assume that the effective open sets $f^{-1}\left(B_{k}^{\prime}\right)$ come with growing enumerations $f^{-1}\left(B_{k}^{\prime}\right)[s]$ such that the predicate $a_{i} \in f^{-1}\left(B_{k}^{\prime}\right)[s]$ is decidable in $i, k, s$ (use the effective basis given by Proposition 2.3.1).

We now proceed to the construction of the sequence $B_{n}[s]$ for each stage $s$. For each $s, B_{n}[s]$ will be a shrinking sequence, $x[s]$ will be defined as the unique member of their intersection and will be one of the points $\left\{a_{j}: j \in \mathbb{N}\right\}$.

Stage 0 . We start with a ball $B_{0}[0]$ of radius $1, B_{n+1}[0]=U_{B_{n}[0]}$ and $\{x[0]\}=$ $\bigcap_{n} B_{n}[0]$. Start with $L_{0}=\emptyset$ and $V_{0}=Y$. Observe that for each $n, B_{n}[0] \cap$ $f^{-1}\left(V_{0}\right)$ is non-empty as it contains $x[0]$.

Stage $s+1$. First, $L_{s+1}$ is obtained by adding to $L_{s}$ all the numbers $k \leq s$ such that $x[s] \in f^{-1}\left(B_{k}^{\prime}\right)[s]$. Let $V_{s+1}$ be the intersection of the open sets $B_{k}^{\prime}$ with $k \in L_{s+1}$.

Let $n=n_{s}$ be the next element enumerated into the halting set. Let $B_{n+1}[s+$ 1] be a ball satisfying $\bar{B}_{n+1}[s+1] \subseteq f^{-1}\left(V_{s+1}\right) \cap B_{n}[s] \backslash \bar{B}_{n+1}[s]$. Such a ball exists: $f^{-1}\left(V_{s+1}\right) \cap B_{n+1}[s]$ is non-empty as it contains $x[s], f$ is irreversible and $B_{n+1}[s]=U_{B_{n}[s]}$. For $n^{\prime} \leq n$, let $B_{n^{\prime}}[s+1]=B_{n^{\prime}}[s]$. For $n^{\prime}>n$ define by induction $B_{n^{\prime}+1}[s+1]=U_{B_{n^{\prime}}[s+1]}$. Let $\{x[s+1]\}=\bigcap_{n} B_{n}[s+1]$.

Verification. By construction one has $\bar{B}_{n+1}[s] \subseteq B_{n}[s]$ and $B_{n+1}[s]=U_{B_{n}[s]}$ 
for sufficiently large $n$ so $B_{n}[s]$ is a shrinking sequence.

We call the settling time of $n$ the minimal number $s$ such that $n_{s^{\prime}} \geq n$ for all $s^{\prime} \geq s$.

We say that $n \in \emptyset^{\prime}$ is a forward element if no element $m<n$ is enumerated into $\emptyset^{\prime}$ after the enumeration stage of $n$ : in other words, the settling time of $n$ coincides with its enumeration stage. As $\emptyset^{\prime}$ is infinite, it has infinitely many forward elements.

Claim 1. For each $n, B_{n}[s]$ is eventually constant.

Proof. Let $s_{0}$ be the settling time of $n: B_{n}[s]=B_{n}\left[s_{0}\right]$ for all $s \geq s_{0}$.

Let $B_{n}$ be its limit. $B_{n}$ is a shrinking sequence as well, let $x$ be the member of its intersection. Observe that the sequence $x[s]$ converges to $x$. Indeed, given $\epsilon$, let $n$ be such that $B_{n}$ has radius $<\epsilon$ and $s_{0}$ be the settling time of $n$ : for all $s \geq s_{0}, x[s] \in B_{n}[s]=B_{n}$ so $d(x[s], x) \leq \epsilon$.

Claim 2. $f(x)$ is computable.

Proof. We prove that a basic open set $B_{k}^{\prime}$ contains $f(x)$ if and only if $k$ is enumerated into the list $L=\bigcup_{s} L_{s}$.

If $k \in L_{s}$ for some $s$, let $n$ be a forward element which is enumerated at some stage $s^{\prime} \geq s . x \in \bar{B}_{n+1}=\bar{B}_{n+1}\left[s^{\prime}+1\right] \subseteq f^{-1}\left(V_{s^{\prime}+1}\right) \subseteq f^{-1}\left(V_{s}\right) \subseteq$ $f^{-1}\left(B_{k}^{\prime}\right)$.

Now let $B_{k}^{\prime}$ be a basic neighborhood of $f(x)$. Let $i_{0}$ be such that $x \in$ $f^{-1}\left(B_{k}^{\prime}\right)\left[i_{0}\right]$. As $x[s]$ converges to $x$ there is $s$ such that $x[s] \in f^{-1}\left(B_{k}^{\prime}\right)\left[i_{0}\right]$ for all $s^{\prime} \geq s$. Let $t=\max \left(s, i_{0}\right): x[t] \in f^{-1}\left(B_{k}^{\prime}\right)\left[i_{0}\right] \subseteq f^{-1}\left(B_{k}^{\prime}\right)[t]$ so $k$ must be added to the list at stage $t+1$ or earlier.

Claim 3. $\emptyset^{\prime}$ is computable relative to $x$.

Proof. Let $\left(p_{i}\right)_{i \in \mathbb{N}}$ be the increasing sequence of forward elements. $\emptyset^{\prime}$ can be computed from the sequence $p_{i}$ and the (computable) enumeration of $\emptyset^{\prime}$.

From $x$ one can inductively compute the sequence $p_{i}$. First, $p_{0}$ is the minimal $n$ such that $x \notin B_{n+1}[0]$. Once $p_{i}$ is known, let $s$ be the stage at which $p_{i}$ is enumerated into $\emptyset^{\prime}$, i.e. $n_{s}=p_{i} \cdot p_{i+1}$ is the minimal $n>p_{i}$ such that $x \notin B_{n+1}[s+1]$.

In the proof $\emptyset^{\prime}$ is encoded into $x$. The argument relativizes: given a set $A \subseteq \mathbb{N}$, there exists $x_{A}$ such that $A$ computes $f\left(x_{A}\right)$ and the pair $\left(x_{A}, A\right)$ computes $A^{\prime}$. All the reductions are uniform, so computing the Turing 
jump operator can be reduced to computing the inverse of $f$ (when $f$ is oneto-one). The notion capturing this idea is Weihrauch reducibility [Wei92, BG11].

Corollary 3.3.1. If $f$ is one-to-one, computable and effectively irreversible then the jump operator is Weihrauch reducible to $f^{-1}$.

\subsection{Application to the ergodic decomposition}

We now present an application of Theorem 3.3.1. Let $P$ be a Borel probability measure $P$ over the Cantor space. $P$ is computable if the real numbers $P[w]$ are uniformly computable. $P$ is shift-invariant if $P[w]=$ $P[0 w]+P[1 w]$ for each finite string $w . P$ is ergodic if it cannot be written as $P=\frac{1}{2}\left(P_{1}+P_{2}\right)$ with $P_{1} \neq P_{2}$ both shift-invariant.

The ergodic decomposition theorem [Phe01] says that every shift-invariant measure can be uniquely decomposed into a convex combination (possibly uncountable) of ergodic measures. Our question is: given a computable shiftinvariant measure, can we compute in a sense its ergodic decomposition? This question was implicitly addressed by V'yugin [V'y97] who constructed a counter example: a countably infinite combination of ergodic measures which is computable but not effectively decomposable. In [Hoy11] we raised the following question: does the ergodic decomposition become computable when restricting to finite combinations? As an application of Theorem 3.3.1, we solve the problem and prove that it is already non-effective in the finite case:

Theorem 3.4.1. There exist two ergodic shift-invariant measures $P$ and $Q$ such that neither $P$ nor $Q$ is computable but $P+Q$ is computable.

The strategy is as follows: the mapping $(P, Q) \mapsto P+Q$ is computable, two-to-one on the space $\mathcal{E} \times \mathcal{E}$ of pairs of ergodic measures and we prove

Theorem 3.4.2. The function $(P, Q) \mapsto P+Q$ defined on $\mathcal{E} \times \mathcal{E}$ is effectively irreversible.

which implies Theorem 3.4.1 by applying Theorem 3.3.1.

Before proving the theorem, we need some preliminaries so show that $\mathcal{E} \times$ $\mathcal{E}$ is an effective Polish space.

We consider the space $\mathcal{P}\left(2^{\mathbb{N}}\right)$ of Borel probability measures over the Cantor space together with the complete metric

$$
d(P, Q)=\sum_{w \in\{0,1\}^{*}} 2^{-|w|}|P[w]-Q[w]| .
$$


The finite rational combinations of Dirac measures are dense in $\mathcal{P}\left(2^{\mathbb{N}}\right)$ and $d$ is computable over them, so $\mathcal{P}\left(2^{\mathbb{N}}\right)$ is an effective Polish space. The subset $\mathcal{I}$ of shift-invariant measures is closed so $d$ is complete over $\mathcal{I}$ as well. $\mathcal{I}$ easily contains a dense computable sequence (take the Markovian measures with rational coefficients), so $\mathcal{I}$ is an effective Polish subspace of $\mathcal{P}\left(2^{\mathbb{N}}\right)$. Let $\mathcal{E} \subseteq \mathcal{I}$ be the set of ergodic shift-invariant measures. The metric $d$ is no longer complete over $\mathcal{E}$, but $\mathcal{E}$ is an effective $G_{\delta^{-}}$set that is c.e., so Proposition 2.3.3 implies that $\mathcal{E}$ is an effective Polish subspace (see [Par61] for results on the Baire category of the set of ergodic measures). We work with the basis given by the intersection of the canonical metric basis of $\mathcal{I}$ with $\mathcal{E}$, which is an effective basis of $\mathcal{E}$.

We now present the proof of Theorem 3.4.2.

Proof of Theorem 3.4.2. Let $B \subseteq \mathcal{I} \times \mathcal{I}$ be an open set and $(P, Q) \in B$ with $P \neq Q$. Let $\epsilon>0$ be such that $d(P, Q)>\epsilon$ and $B(P, \epsilon) \times B(Q, \epsilon) \subseteq B$. Let $\delta=\epsilon / 4$ and $U_{B}=B(P, \delta) \times B(Q, \delta) \subseteq B$. Observe that $U_{B}$ can be effectively obtained from $B$.

We now show that if $V \subseteq \mathcal{I}$ is open and $f^{-1}(V)$ intersects $B$ then it intersects $B \backslash U_{B}$, where $f: \mathcal{E} \times \mathcal{E} \rightarrow \mathcal{I}$ is the sum mapping. Let $\left(P_{1}, Q_{1}\right) \in$ $f^{-1}(V) \cap B$. If $\left(P_{1}, Q_{1}\right) \notin U_{B}$ then we are done. Assume then that $\left(P_{1}, Q_{1}\right) \in$ $U_{B}$, which implies $d\left(P_{1}, Q_{1}\right)>2 \delta$. For $\lambda \in[0,1]$, define

$$
\begin{aligned}
P_{\lambda} & =\lambda P_{1}+(1-\lambda) Q_{1}, \\
Q_{\lambda} & =\lambda Q_{1}+(1-\lambda) P_{1} .
\end{aligned}
$$

First observe that $P_{\lambda}+Q_{\lambda}=P_{1}+Q_{1} \in V$. There is some $\lambda \in(0,1)$ such that $\left(P_{\lambda}, Q_{\lambda}\right) \in B \backslash U_{B}$, more precisely $\delta<d\left(P, P_{\lambda}\right)<\epsilon$ and $\delta<d\left(Q, Q_{\lambda}\right)<$ $\epsilon$. Indeed,

$$
d\left(P_{1}, P_{\lambda}\right)=d\left(Q_{1}, Q_{\lambda}\right)=(1-\lambda) d\left(P_{1}, Q_{1}\right) .
$$

and as $d\left(P_{1}, Q_{1}\right)>2 \delta$ there exists $\lambda \in(0,1)$ such that $(1-\lambda) d\left(P_{1}, Q_{1}\right)=2 \delta$. One then has

$$
d\left(P, P_{\lambda}\right) \leq d\left(P, P_{1}\right)+d\left(P_{1}, P_{\lambda}\right)<3 \delta<\epsilon
$$

and

$$
d\left(P, P_{\lambda}\right) \geq d\left(P_{1}, P_{\lambda}\right)-d\left(P, P_{1}\right)>\delta
$$

and similarly $\delta<d\left(Q, Q_{\lambda}\right)<\epsilon$. Observe that the shift-invariant measures $P_{\lambda}$ and $Q_{\lambda}$ are not ergodic. However the ergodic measures are dense in 
the set of shift-invariant measures so there exist two ergodic measures $P^{\prime}, Q^{\prime}$ close to $P_{\lambda}$ and $Q_{\lambda}$ respectively, such that

$$
\begin{gathered}
\delta<d\left(P, P^{\prime}\right)<\epsilon, \\
\delta<d\left(Q, Q^{\prime}\right)<\epsilon, \\
P^{\prime}+Q^{\prime} \in V
\end{gathered}
$$

which implies that $\left(P^{\prime}, Q^{\prime}\right) \in f^{-1}(V) \cap B \backslash U_{B}$, which is then non-empty.

\section{Directional genericity}

Given an effectively irreversible function $f$,

- Theorem 3.2.1 tells us that if $x$ is 1-generic then $x$ is not computable relative to $f(x)$,

- Theorem 3.3.1 tells us that there exist non-computable $x$ such that $f(x)$ is computable.

The two results are "disjoint" in the sense that in general a single $x$ cannot at the same time be 1-generic and have a computable image, except for some particular functions like constant functions. We raise the following question: is it possible to bring the two results closer together? How far can $x$ be from being computable, given that $f(x)$ is computable? How generic can $x$ be? In this section we give an answer to these questions, introducing a notion of genericity that is compatible with a weak form of computability.

For the sake of simplicity, we will assume that $f$ is the identity. We fix a set $X$, endowed with an effective Polish topology $\tau$ and a weaker effective topology $\tau^{\prime}$. In doing so we lose no generality, as a function $f:\left(X, \tau_{X}\right) \rightarrow$ $\left(Y, \tau_{Y}\right)$ can always be thought as the identity from $\left(X, \tau_{X}\right)$ to $\left(X, \tau^{\prime}\right)$ where $\tau^{\prime}$ is the initial topology of $f$ whose open sets are the preimages of $\tau_{Y^{-}}$open sets.

\subsection{Being generic from above}

Let $(X, \tau)$ be an effective Polish space and $\tau^{\prime}$ be an effective topology on $X$ that is effectively weaker than $\tau$ : the basic $\tau^{\prime}$-open sets are effective $\tau$-open sets, uniformly. In other words, we require the identity function from $(X, \tau)$ to $\left(X, \tau^{\prime}\right)$ to be computable.

Our general goal is to build elements of $X$ that are to some extent generic in the topology $\tau$ but still computable in the topology $\tau^{\prime}$. The 
latter condition is usually weaker than being computable in the topology $\tau$, and will be our weak notion of computability. We now have to define a suitable notion of genericity.

The topology $\tau^{\prime}$ induces a pre-order on $X$, called the specialization preorder $\leq$ :

$$
x \leq y \Longleftrightarrow \text { every } \tau^{\prime} \text {-neighborhood of } x \text { is a } \tau^{\prime} \text {-neighborhood of } y \text {. }
$$

$x \leq y$ means that if one describes $x$ by listing its basic neighborhoods then one can never distinguish $x$ from $y$. Observe that when $\tau^{\prime}$ is $T_{0}, \leq$ is an order, and when $\tau^{\prime}$ is $T_{1}$, $\leq$ is the trivial ordering (equality).

Definition 4.1. To $x \in X$ we associate

$$
S_{x}=\{y \in X: x \leq y\}
$$

which is the intersection of all the $\tau^{\prime}$-neighborhoods of $x$.

$S_{x}$ is the set of elements that cannot be distinguished from $x$ when describing $x$ in the topology $\tau^{\prime}$. If $\tau^{\prime}$ is $T_{1}$ then $S_{x}=\{x\}$ for all $x$.

In a game where the player describes an element $x$ in the $\tau^{\prime}$-topology, the player enumerates the basic $\tau^{\prime}$-neighborhoods of $x$. Each enumerated basic open set is a commitment: if $V$ is enumerated then $x$ must belong to $V$. Each commitment reduces the degrees of freedom of the player to fool the opponent. However some free space is always left, and this space is precisely $S_{x}$ : at any moment the player is allowed to move into $S_{x}$ (and then change $x$ at the same time). As a consequence, during the computation of $x$ in the topology $\tau^{\prime}$, the player is able to make $x$ as generic as possible, inside the subset $S_{x}$. This motivates the following definition.

Definition 4.2. Let $(X, \tau)$ be an effective Polish space, $A \subseteq X$ and $x \in A$. We say that $x$ is generic inside $A$ if for every effective open set $U \subseteq X$,

- either $x \in U$,

- or there exists a neighborhood $B$ of $x$ such that $B \cap U \cap A=\emptyset$.

If $\tau^{\prime}$ is a weaker topology on $X$ then we say that $x$ is generic from above if $x$ is generic inside $S_{x}=\{y \in X: x \leq y\}$, where $\leq$ is the specialization pre-order induced by $\tau^{\prime}$.

Every 1-generic element is generic inside any set containing it. Let us give a few examples illustrating these notions. For $A=X$, being generic 
inside $A$ is the same as being 1-generic. Every element $x$ is vacuously generic inside $\{x\}$. In the product space $X \times X,(x, y)$ is generic in $\{x\} \times X$ if and only if $y$ is 1 -generic relative to $x$, i.e. $y$ does not belong to the boundary of any open set that is effective relative to $x$ (effective open subsets of $X$ relative to $x$ are the same as the sections at $x$ of effective open subsets of $X \times X)$.

Informally, $x$ is generic from above means that $x$ belongs to every effective open set that is dense above it, for the specialization order induced by $\tau^{\prime}$ (while a 1-generic elements belongs to every effective open set that is dense along it).

With this notion in hand we obtain the sought combination of Theorems 3.2.1 and 3.3.1. For this we need a reasonable technical assumption on the bases $\mathcal{B}$ and $\mathcal{B}^{\prime}$ of the topologies $\tau$ and $\tau^{\prime}$ respectively.

Assumption 1 There is an algorithm that given a finite number of basic open sets from $\mathcal{B}$ and $\mathcal{B}^{\prime}$, decides whether their intersection is nonempty, where $\mathcal{B}$ is a basis associated with a complete effective metric.

In practice this assumption is often satisfied. We do not know how to prove the next theorem without this assumption, and we do not know whether the theorem fails without this assumption.

Theorem 4.1.1 (Generic and weakly computable). Let $(X, \tau)$ be an effective Polish space and $\tau^{\prime}$ and effectively weaker topology, satisfying assumption 1. Let $U_{n}$ be uniformly effective dense $\tau$-open sets. There exists $x \in \bigcap_{n} U_{n}$ such that

- $x$ is generic inside $S_{x}$,

- $x$ is $\tau^{\prime}$-computable.

Observe that the theorem is only interesting when $\tau^{\prime}$ is not $T_{1}$, otherwise $S_{x}=\{x\}$ and the first condition is vacuously satisfied for every $x$.

Observe that $x$ is not in general weakly 1 -generic, so it does not belong to every dense effective open set. However, if an effective sequence of such sets $U_{n}$ is given in advance then $x$ can be taken in their intersection, as stated by the theorem. This is possible as the family of dense effective open sets is not enumerable in general.

Proof. Let $a_{i}$ be a dense computable sequence of simple points in $(X, \tau)$. Again we can assume w.l.o.g. that the basic open sets $B_{k}^{\prime} \in \mathcal{B}^{\prime}$ come with a 
computable enumeration $B_{k}^{\prime}[s]$ ( $B_{k}^{\prime}[s]$ is an effective open set) such that the predicate $a_{i} \in B_{k}^{\prime}[s]$ is decidable in $i, k, s$ (using Proposition 2.3.1).

The proof is a finite injury argument. We want to satisfy the requirements

$$
R_{e}: x \in W_{e} \text { or } \exists \epsilon, B(x, \epsilon) \cap W_{e} \cap S_{x}=\emptyset,
$$

where $W_{e}$ is the effective open set with number $e$ (in the sequel, $W_{e, s}$ will be computable growing finite unions of elements of $\mathcal{B}$ with union $W_{e}$ ). At stage $s$, each requirement $R_{e}$ is assigned a ball $B_{e}[s]$. They satisfy $\bar{B}_{e+1}[s] \subseteq$ $B_{e}[s] \cap U_{e}$. For each $e$, the sequence $B_{e}[s]$ is eventually constant when $s$ grows. At the same time, a list $L \subseteq \mathbb{N}$ is enumerated containing exactly the indices of the basic $\mathcal{B}^{\prime}$-neighborhoods of $x . L$ is obtained as the union of a growing computable sequence of finite sets $L_{s}$. We denote by $V_{s}$ the finite intersection of elements of $\mathcal{B}^{\prime}$ whose indices are given by $L_{s}$, i.e. $V_{s}=$ $\bigcap_{k \in L_{s}} B_{k}^{\prime} . V_{s}$ will be a neighborhood of $x$ in the topology $\tau^{\prime}$. In order to satisfy the requirement $R_{e}$, one tests whether $B_{e}[s]$ intersects $W_{e, s} \cap V_{s}$ and if it is so, forces $x$ to belong to the intersection.

Stage 0 . Let $B_{0}[0]$ be any ball of radius $<2^{-0}$ and inductively choose $\bar{B}_{e+1}[0] \subseteq$ $B_{e}[0] \cap U_{e}$ of radius $<2^{-e-1}$. Let $x_{0}$ be the center of $B_{0}[0]$ and $L_{0}=\emptyset$.

Stage $s+1$. Let $e \leq s$ be minimal such that $B_{e}[s] \cap W_{e, s} \cap V_{s} \neq \emptyset$ (decidable by Assumption 1) and $R_{e}$ is not already declared satisfied, if it exists (decidable property). Let $B_{e^{\prime}}[s+1]=B_{e^{\prime}}[s]$ for $e^{\prime} \leq e$, let $\bar{B}_{e+1}[s+1] \subseteq$ $B_{e}[s] \cap W_{e, s} \cap V_{s} \cap U_{e}$ have radius $<2^{-e-1}$ and $x_{s+1}$ be the center of $B_{e+1}[s+1]$. Define inductively $\bar{B}_{e^{\prime}+1}[s+1] \subseteq B_{e^{\prime}}[s+1] \cap U_{e^{\prime}}$ of radius $<2^{-e^{\prime}-1}$ for $e^{\prime}>e$. We say that $R_{e}$ acts and $R_{e}$ is declared satisfied. All $R_{e^{\prime}}$ with $e^{\prime}>e$ are initialized, which means that all of them are declared unsatisfied.

If such an $e \leq s$ does not exist then let $B_{e}[s+1]=B_{e}[s]$ for all $e$ and $x_{s+1}=x_{s}$.

Let $L_{s+1}$ contain $L_{s}$ together with every $k \leq s$ such that $x_{s+1} \in B_{k}^{\prime}[s]$.

Verification. By the usual analysis of finite injury arguments, each requirement acts finitely many times, so for each $e$ there is $s_{0}$ such that $B_{e}[s]=$ $B_{e}\left[s_{0}\right]$ for all $s \geq s_{0}$. Let $B_{e}=B_{e}\left[s_{0}\right]$. One has $\bar{B}_{e+1} \subseteq B_{e}$ and $B_{e}$ has radius $<2^{-e}$. Let $x$ be the unique member of $\bigcap_{e} B_{e}$.

Claim 4. The sequence $x_{s}$ converges to $x$.

For each $k$, and all sufficiently large $s$, only requirements $R_{e}$ with $e>k$ act, so $x_{s+1} \in B_{e+1}[s+1] \subseteq B_{k}[s+1]=B_{k}$. As $x \in B_{k}$ and $B_{k}$ has radius $<2^{-k}, d\left(x_{s+1}, x\right)<2^{-k+1}$ for all sufficiently large $s$. 
Claim 5. $x \in \bigcap_{e} U_{e}$.

By construction, $B_{e+1} \subseteq U_{e}$ for all $e$, hence $x \in \bigcap_{e} B_{e+1} \subseteq \bigcap_{e} U_{e}$.

Claim 6. $L$ lists exactly the elements of $\mathcal{B}^{\prime}$ containing $x$, hence $x$ is $\tau^{\prime}$ computable.

First, $x$ belongs to each $V_{s}$ by construction of the balls $B_{e}[s]$, so $L$ lists only (indices of) neighborhoods of $x$. Conversely, assume that $B_{k}^{\prime}$ is a neighborhood of $x$. It implies that some $B_{k}^{\prime}\left[s_{0}\right]$ is a neighborhood of $x$. As $x_{s}$ converges to $x, x_{s}$ belongs to $B_{k}^{\prime}\left[s_{0}\right]$ for all $s$ larger that some $s_{1}$. As a result, for $s>\max \left(s_{0}, s_{1}\right), k$ is listed in $L_{s}$.

Claim 7. $x$ is generic inside $S_{x}$.

Let $e \in \mathbb{N}$ be such that $x \notin W_{e}$. Let $s$ be such that no requirement $e^{\prime}<e$ acts from stage $s$ on. $R_{e}$ cannot act at a stage $s^{\prime} \geq s$, otherwise $x \in B_{e+1}=$ $B_{e+1}\left[s^{\prime}+1\right] \subseteq W_{e}$ which contradicts the assumption $x \notin W_{e}$. In the same way, $R_{e}$ cannot be declared satisfied at stage $s$, otherwise $x \in B_{e+1}=$ $B_{e+1}\left[s^{\prime}+1\right] \subseteq W_{e}$. As $R_{e}$ never acts after stage $s$, it means that $x \in$ $B_{e}=B_{e}[s]$ and $B_{e}[s] \cap W_{e} \cap S_{x}=\emptyset$, otherwise there exists $s^{\prime} \geq s$ such that $B_{e}[s] \cap W_{e, s^{\prime}} \cap S_{x} \neq \emptyset$, hence $B_{e}\left[s^{\prime}\right] \cap W_{e, s^{\prime}} \cap V_{s^{\prime}} \neq \emptyset$ as $B_{e}\left[s^{\prime}\right]=B_{e}[s]$ and $S_{x} \subseteq V_{s^{\prime}}$, and then $R_{e}$ must act at stage $s^{\prime}$ as it is not declared satisfied. This is a contradiction.

The point $x$ provided by Theorem 4.1 .1 actually satisfies a stronger notion of genericity than being generic inside $S_{x}$.

Lemma 4.1.1. For each effective open set $W_{e}=\bigcup_{s} W_{e}[s]$,

- either $x \in W_{e}$,

- or there exists a neighborhood $B$ of $x$ such that $B \cap\left(\bigcup_{s} W_{e}[s] \cap V_{s}\right)=\emptyset$.

Proof. Indeed, if $x \notin W_{e}$ then when all $R_{e^{\prime}}$ with $e^{\prime} \leq e$ have settled, $B_{e}[s]=$ $B_{e}$ and $B_{e}[s] \cap W_{e}[s] \cap V_{s}=\emptyset$ otherwise $R_{e}$ will act and force $x$ to fall into $W_{e}$, so one can take $B=B_{e}$.

In other words, $x$ satisfies the condition of 1-genericity not for every effective open set $W_{e}$, but for the effective open set $\bigcup_{s} W_{e}[s] \cap V_{s}$. This genericity condition has the consequence that $x$ behaves in some respects as a 1-generic point, as illustrated by the following two results.

Corollary 4.1.1. The point $x$ provided by Theorem 4.1 .1 is low, i.e. the set $\left\{e \in \mathbb{N}: x \in W_{e}\right\}$ is $\Delta_{2}^{0}$ (or $\emptyset^{\prime}$-computable, or limit of a computable sequence). 
Proof. For each $e$, the computable predicate $B_{e}[s] \cap W_{e}[s] \cap V_{s} \neq \emptyset$ converges to the predicate $x \in W_{e}$. Indeed, if $B_{e}[s] \cap W_{e}[s] \cap V_{s} \neq \emptyset$ for infinitely many $s$ then $R_{e}$ will eventually act and never be injured later, so $x$ will be forced to fall into $W_{e}$. If $x \in W_{e}$ then for sufficiently large $s, B_{e}[s]=B_{e}$ and $x \in W_{e}[s]$ so $B_{e}[s] \cap W_{e}[s] \cap V_{s} \neq \emptyset$.

On compact spaces satisfying assumption 1, Theorem 4.1 .1 is indeed a strengthening of Theorem 3.3.1.

Corollary 4.1.2. Assume that $(X, \tau)$ is compact. If id : $(X, \tau) \rightarrow\left(X, \tau^{\prime}\right)$ is effectively irreversible then the point $x$ provided by Theorem 4.1 .1 can be taken to be non-computable.

Proof. One can take $x$ in the dense effective $G_{\delta}$-set given by Proposition 3.2 .1 , so that id is not locally continuously invertible at $x$.

If $x$ is computable then there exists $e$ such that $W_{e}=X \backslash\{x\}$. As $x \notin W_{e}$ there exists a neighborhood $B$ of $x$ such that $B \cap \bigcup_{s} W_{e}[s] \cap V_{s}=\emptyset$. Let $\bar{U}_{B} \subseteq$ $B$ come from the local continuous non-invertibility of $i d$ at $x$. As $W_{e}$ covers the compact set $X \backslash U_{B}$, there exists $s$ such that $W_{e}[s]$ already covers that set. As $V_{s}$ is a $\tau^{\prime}$-neighborhood of $x, B \cap V_{s} \backslash U_{B} \neq \emptyset$ so $B \cap V_{s} \cap W_{e}[s] \neq \emptyset$, which contradicts the choice of $B$.

We now illustrate directional genericity in several situations and show how Theorem 4.1.1 embodies many constructions encountered in computability theory. It means that in many situations, in order to construct an object satisfying a given set of requirements, one only has to find the suitable topologies $\tau$ and $\tau^{\prime}$ that make directionally generic objects have the sought properties. Theorem 4.1 .1 can then be directly applied, instead of explicitly constructing the object by means of a finite injury argument.

\subsection{Genericity for c.e. sets}

We consider the Cantor space $X$ of subsets of $\mathbb{N}$. Here $\tau$ is the Cantor topology and $\tau^{\prime}$ is the Scott topology generated by the sets $\{A \subseteq \mathbb{N}: F \subseteq A\}$, where $F$ ranges among the finite subsets of $\mathbb{N}$. For a set $A \subseteq \mathbb{N}, S_{A}=\{B \subseteq$ $\mathbb{N}: A \subseteq B\}$ is the class of supersets of $A$.

Definition 4.3. A set $A \subseteq \mathbb{N}$ is generic from above if it is 1-generic inside $S_{A}$, which means that for every effective open class $\mathcal{U}$, either $A \in \mathcal{U}$ or there exists $n$ such that $\left[A \uparrow_{n}\right] \cap \mathcal{U} \cap S_{A}=\emptyset$. 
In other words, $A$ is generic from above if it belongs to every effective open class that is dense above $A$ in the subset ordering. This should be compared to the notion of 1-generic set, which belongs to every effective open class that is dense along or at it. Observe that a co-finite set $A$ is vacuously generic from above as there is an $n$ such that $\left[A \uparrow_{n}\right] \cap S_{A}=\{A\}$. Hence only co-infinite sets that are generic from above are interesting.

As a direct application of Theorem 4.1.1 we obtain:

Corollary 4.2.1. There exists a co-infinite c.e. set $A \subseteq \mathbb{N}$ that is generic from above.

Proof. The class of co-infinite sets is a dense effective $G_{\delta}$-set.

As the next result shows, Theorem 4.1.1 embodies simple finite injury arguments such as the Friedberg-Muchnik theorem for instance. Interestingly many arguments showing that 1-generic sets satisfy some property already apply to sets that are generic from above. Indeed, in these arguments, an effective open class is shown to be dense at the set and it often happens that it is even dense above the set. We now give illustrations of this phenomenon.

Proposition 4.2.1. Let $A$ be co-infinite and generic from above. $\mathbb{N} \backslash A$ is hyperimmune, $A=A_{1} \oplus A_{2}$ where $A_{1}$ and $A_{2}$ are Turing incomparable, $A$ is not autoreducible.

Proof. The simple arguments showing the results for 1-generic sets actually give this stronger result, observing that the involved open set is not only dense along $A$, but even above $A$. For instance, to prove that $A_{2} \Varangle_{T} A_{1}$, given a Turing functional $\phi$, let $U=\left\{A_{1} \oplus A_{2}: \exists n, \phi^{A_{1}}(n)=0 \wedge A_{2}(n)=\right.$ 1\}. If $\phi^{A_{1}}=A_{2}$ then replacing a 0 in $A_{2}$ by a 1 arbitrarily far gives an element of $U$ arbitrarily close to $A_{1} \oplus A_{2}$ that is above (i.e. is a superset of) $A_{1} \oplus A_{2}$.

It happens that the co-infinite sets that are generic from above are exactly the $p$-generic sets defined by Ingrassia [Ing81].

\subsection{Genericity for left-c.e. reals}

We consider the unit real interval [0,1]. $\tau$ is the Euclidean topology, $\tau^{\prime}$ is the topology induced by the semi-lines $(x, 1]$. The specialization order is the natural ordering on real numbers. For a real $x \in[0,1], S_{x}=[x, 1]$.

Definition 4.4. A real $x \in[0,1]$ is generic from the right if it is 1 generic inside $[x, 1]$, which means that for every effective open set $\mathcal{U} \subseteq[0,1]$, either $x \in \mathcal{U}$ or there exists $\epsilon>0$ such that $[x, x+\epsilon) \cap \mathcal{U}=\emptyset$. 
Again $x$ is generic from the right if it belongs to every effective open set that is dense above $x$ in the real ordering.

Kurtz built a left-c.e. weakly 1-generic real (see Theorem 1.8.49 in [Nie09]). The construction even gives a left-c.e. real that is generic from the right. One can think of the proof of Theorem 4.1.1 as a kind of generalization of this argument (replacing the lexicographic order used in the proof appearing in [Nie09] by the specialization pre-order induced by $\tau^{\prime}$ ).

Genericity from the right easily lies between two classical notions of genericity.

Proposition 4.3.1. Every 1-generic is generic from the right. Every generic from the right is weakly-1-generic. The implications are strict.

Proof. An open set that is dense is dense on the right of $x$. An open set that is dense on the right of $x$ is dense along $x$. Right-c.e. reals cannot be generic on the right, but there exists a right-c.e. weakly-1-generic real. Leftc.e. reals cannot be 1-generic, but there exists a left-c.e. real that is generic on the right.

In Section 4.5 we will separate genericity from the right from weakly-1genericity among the left-c.e. reals.

Solovay reducibility vs. cl-reducibility. If $A$ is a subset of $\mathbb{N}$ then we denote by $x_{A}$ the real number whose binary expansion is $A$. In [DHL04] it is proved that there exist two sets $A, B$ such that $A$ is a c.e. set, $x_{B}$ is a left-c.e. real and $A \leq_{\mathrm{cl}} B$ but $x_{A} \not_{\mathrm{S}} x_{B}$. Here $\leq_{\mathrm{cl}}$ stands for computably Lipschitz reducibility and $\leq_{\mathrm{S}}$ stand for Solovay reducibility. The construction is a finite injury argument, which again is captured by Theorem 4.1.1.

Let us recall that $A \leq_{\mathrm{cl}} B$ means that there is a Turing functional computing $A$ with oracle $B$, reading the first $n+c$ bits of $B$ to compute the $n$ first bits of $A$, for some constant $c$ and all $n . x_{A} \leq_{\mathrm{S}} x_{B}$ means that there exists a constant $c \in \mathbb{N}$ and computable sequences $a_{i} \nearrow x_{A}$ and $b_{i} \nearrow x_{B}$ such that $x_{A}-a_{i} \leq c\left(x_{B}-b_{i}\right)$ for all $i$, or equivalently that $c x_{B}-x_{A}$ is left-c.e.

Theorem 4.3.1. Let $x_{B}$ be left-c.e. and generic on the right and $A=\{w \in$ $\left.2^{<\mathbb{N}}: w<_{\text {lex }} B\right\}$. One has $A \leq_{c l} B$ but $x_{A} \not_{S} x_{B}$.

Note that we identify $A$ with a subset of $\mathbb{N}$ by using a computable bijection between $2^{<\mathbb{N}}$ and $\mathbb{N}$ (we will assume that the string represented by a number $n$ has length at most $n$ ). 
Proof. The reduction $A \leq_{\mathrm{cl}} B$ is obvious: to know whether $w<_{\text {lex }} B$, one only needs to know the $|w|$ first bits of $B$.

Assume that $x_{A} \leq_{\mathrm{S}} x_{B}$. It implies the existence of a one-to-one computable enumeration $w_{i}$ of $A$, a computable sequence $b_{i} \nearrow x_{B}$ and a computable order $h: \mathbb{N} \rightarrow \mathbb{N}$ such that for all $i \in \mathbb{N}$, if $x_{B}-b_{i}<2^{-h(n)}$ then $\left|w_{i}\right|>n$. Let $B_{i}$ be the maximal element of $\left\{w_{0}, \ldots, w_{i}\right\}$ in the lexicographic ordering. If $x_{B}-b_{i}<2^{-h(n)}$ then $B_{i} \uparrow_{n}=B \uparrow_{n}$ : indeed, the string $B \uparrow_{n}$ belongs to $A$ so it must be $w_{j}$ for some $j$, which must be less than $i$.

For $w \in 2^{<\mathbb{N}}$, let $[w]$ be the interval containing all real numbers having a binary expansion starting with $w$, namely $[w]=\left[0 . w, 0 . w+2^{-|w|}\right]$.

Let $U=\bigcup_{n, i}\left(b_{i}, b_{i}+2^{-h(n)}\right) \backslash\left[B_{i} \uparrow_{n}\right]$. $U$ is an effective open set. $U$ does not contain $x_{B}$. Indeed, if $x_{B}-b_{i}<2^{-h(n)}$ then $B_{i} \uparrow_{n}=B \uparrow_{n}$ so $x_{B} \in\left[B_{i} \uparrow_{n}\right]$. We now prove that $U$ is dense on the right of $x_{B}$, which contradicts the assumption that $x_{B}$ is generic on the right. As $x_{B}$ is generic on the right it is weakly 1-generic, so there exist infinitely many $n$ such that $B$ contains all the natural numbers from $n$ to $h(n)$. In other words for infinitely many $n, x_{B}$ is very close to the right endpoint of $\left[\left.B\right|_{n}\right]$, namely at distance $<2^{-h(n)}$. For such $n$ and sufficiently large $i,\left(b_{i}, b_{i}+2^{-h(n)}\right) \backslash\left[B_{i} \uparrow_{n}\right]$ is a non-empty subset of the interval $\left(x_{B}, x_{B}+2^{-h(n)}\right)$.

We actually prove more: there is no computable order $h$ and no computable sequences $a_{i} \nearrow x_{A}, b_{i} \nearrow x_{B}$ such that $x_{B}-b_{i} \leq 2^{-h(n)}$ implies $x_{A}-a_{i} \leq 2^{-n}$, which would be a generalization of Solovay reducibility.

Left-c.e. reals with only computable presentations. Downey and LaForte [DL02] proved the existence of non-computable left-c.e. reals $x$ all of whose presentations are computable: each prefix-free c.e. set $A$ of finite binary strings satisfying $\sum_{w \in A} 2^{-|w|}=x$ is actually a computable set. A corollary of a result of Stephan and Wu [SW05] is that any such real is weakly 1-random, i.e. it must belong to every effective open set of measure one. Actually it must be weakly-1-generic and even generic from the right.

Proposition 4.3.2. If $x$ is a non-computable left-c.e. real all of whose presentations are computable then $x$ is generic from the right.

Proof. Let $U$ be an effective open set that does not contain $x$ : we must find $y>x$ such that $[x, y)$ is disjoint from $U$. First replace $U$ by $V=U \cup$ $[0, x)$. Let $A$ be a prefix-free c.e. set such that $V=\bigcup_{w \in A}[w]$. The set $A_{<x}=$ $\left\{w \in A: w<_{\operatorname{lex}} x\right\}$ is a presentation of $x$ hence it is computable, so $A_{>x}=$ $\left\{w \in A: w>_{\text {lex }} x\right\}=A \backslash A_{<x}$ is c.e. As a result, $y:=\inf \bigcup_{w \in A_{>x}}[w]$ is 
right-c.e. As $x$ is not computable and $x \leq y$, one has $x<y$ and we get the result as $[x, y)$ is disjoint from $U$.

\subsection{Genericity for $\Pi_{1}^{0}$-classes}

We work on the set $\mathrm{CL}\left(2^{\mathbb{N}}\right)$ of non-empty closed subsets of the Cantor space, endowed with the so-called hit-or-miss topology $\tau_{h m}$ [Mic51]. $\tau_{h m}$ is generated by the miss sets $\mathcal{U}_{u}=\left\{P \in \operatorname{CL}\left(2^{\mathbb{N}}\right): P \cap[u]=\emptyset\right\}$ where $u \in 2^{<\mathbb{N}}$, together with their complements (the hit sets). We obtain an effective Polish space $\left(\mathrm{CL}\left(2^{\mathbb{N}}\right), \tau_{h m}\right)$. A computable element of this space is usually called a computable closed set, and is the set of infinite branches of a computable tree without dead-ends.

Proposition 4.4.1. In the space $\left(C L\left(2^{\mathbb{N}}\right), \tau_{h m}\right)$, every weakly-1-generic element contains only weakly-1-generic sequences.

Proof. Let $U \subseteq 2^{\mathbb{N}}$ be a dense effective open set. Let $\mathcal{U}=\left\{P \in \operatorname{CL}\left(2^{\mathbb{N}}\right)\right.$ : $P \subseteq U\} . \mathcal{U}$ is a dense effective open set in the space $\operatorname{CL}\left(2^{\mathbb{N}}\right)$. Hence every weakly-1-generic closed set $P$ belongs to $\mathcal{U}$, i.e. is contained in $U$.

We consider a weaker topology $\tau_{m}$ called the miss topology, generated by the miss sets $\mathcal{U}_{u}$ with $u \in 2^{<\mathbb{N}}$. A $\Pi_{1}^{0}$-class is a computable member of $\left(\mathrm{CL}\left(2^{\mathbb{N}}\right), \tau_{m}\right)$. The specialization pre-order induced by $\tau_{m}$ is the reverse inclusion, so that for each non-empty closed set $P$ one has $\mathcal{S}_{P}=\{Q \in$ $\left.\mathrm{CL}\left(2^{\mathbb{N}}\right): Q \subseteq P\right\}$ (being "above" $P$ in this pre-order means being inside $P$ ). Definition 4.2 is instantiated as follows.

Definition 4.5. A non-empty closed set $P \subseteq 2^{\mathbb{N}}$ is generic from inside if $P$ is 1-generic inside $S_{P}$, which means that for every effective $\tau_{h m}$-open set $\mathcal{U} \subseteq \mathrm{CL}\left(2^{\mathbb{N}}\right)$, either $P \in \mathcal{U}$ or there exists a $\tau_{h m}$-neighborhood $\mathcal{N}$ of $P$ such that $\mathcal{N} \cap \mathcal{U} \cap \mathcal{S}_{P}=\emptyset$.

Proposition 4.4.2. Every closed set $P$ that is generic from inside has empty interior, i.e. has a dense complement.

Proof. Given a cylinder $[u]$, the class $\mathcal{U}$ of non-empty closet sets that do not contain $[u]$ is an effective open class: it is the union over $[v] \subseteq[u]$ of the miss sets $\mathcal{U}_{v}$. If $[v] \subseteq[u]$ then $P \backslash[v]$ belongs to $\mathcal{S}_{P} \cap \mathcal{U}$ and is arbitrarily close to $P$ in the topology $\tau_{h m}$ as the length of $v$ tends to infinity. As $P$ is generic from inside, $P$ must belong to $\mathcal{U}$ so $P$ does not contain $[u]$.

As a result, no member of a $\Pi_{1}^{0}$-class $P$ that is generic from inside can be weakly-1-generic. However all the elements of $P$ are weakly-1-generic 
inside $P$ : if $U$ is an effective open subset of the Cantor space such that $P \cap U$ is dense in $P$ then $U$ contains every member of $P$, i.e. $U$ contains $P$.

Proposition 4.4.3. If $P$ is generic from inside then every member of $P$ is weakly-1-generic inside $P$.

Proof. Let $U \subseteq 2^{\mathbb{N}}$ be an effective open set. Consider the set $\mathcal{U}=\{P$ : $P \subseteq U\} . \mathcal{U}$ is an effective open set in the space $\left(\mathrm{CL}\left(2^{\mathbb{N}}\right), \tau_{h m}\right)$ (and even in the topology $\left.\tau_{m}\right)$. If $P \cap U$ is dense in $P$ then there exists $Q \subseteq P \cap U$ arbitrarily $\tau_{h m^{-}}$-close to $P$ (let $Q$ be a finite set of points from $P \cap U$ whose $\epsilon$ neighborhood covers $P$, for arbitrarily small $\epsilon$ ), so $P$ belongs to the closure of $\mathcal{U} \cap \mathcal{S}_{P}$. If $P$ is generic from inside then $P$ must belong to $\mathcal{U}$, so $P \subseteq U$.

In particular,

Corollary 4.4.1. A perfect closed set that is generic from inside has no computable member.

Proof. If $x$ is computable then $U=2^{\mathbb{N}} \backslash\{x\}$ is an effective open set. If $P$ has no isolated point then $P \cap U$ is dense in $P$. By the previous result, $P$ is then contained in $U$, i.e. $P$ does not contain $x$.

Now, Theorem 4.1.1 can be instantiated as follows.

Corollary 4.4.2. There exists a perfect $\Pi_{1}^{0}$-class that is generic from inside.

Proof. Being perfect, or having no isolated point is a dense effective $G_{\delta^{-}}$ property in the space $\left(\mathrm{CL}\left(2^{\mathbb{N}}\right), \tau_{h m}\right)$.

\subsection{Genericity for regular $\Pi_{1}^{0}$-classes}

We know from Proposition 4.3.1 that every real $x \in[0,1]$ that is generic on the right is weakly-1-generic, but not the converse. Here we prove the existence of left-c.e. reals that are weakly-1-generic but not generic from the right.

To this end we need to construct a $\Pi_{1}^{0}$-set $P$ such that (i) its leftmost element $x$ is weakly-1-generic, and (ii) the complement of $P$ is dense on the right of $x$. The first condition requires the class to have non-empty interior, and even that the interior of $P$ be dense along $x$. Together with the second condition, it implies that $x$ should not be isolated in the boundary of $P$.

The class $P$ that we build will actually satisfy these conditions at every point of its boundary: $P$ is regular (it coincides with the closure of its 
interior), its boundary is perfect (has no isolated point) and contains only weakly-1-generic points.

A suitable way of describing a regular closed set $C$ is by giving approximations of $C$ in the hit-or-miss topology and at the same time enumerating its interior. This can be formalized by introducing a new topology $\tau$ on $\mathrm{CL}([0,1])$ that is stronger than the hit-or-miss topology $\tau_{\mathrm{hm}}$. First, the hit-or-miss topology $\tau_{\mathrm{hm}}$ is generated by the hit sets $\{C \in \mathrm{CL}([0,1])$ : $C \cap(a, b) \neq \emptyset\}$ where $a<b$ are rational, and the miss sets $\{C \in \mathrm{CL}([0,1])$ : $C \cap[a, b]=\emptyset\}$ where $a<b$ are again rational. The stronger topology $\tau$ is generated by the hit-or-miss open sets together with the sets

$$
\{C \in \mathrm{CL}([0,1]):[a, b] \subseteq \operatorname{int}(C)\},
$$

where $a<b$ are rational numbers and $\operatorname{int}(C)$ is the interior of $C$. A canonical enumeration of the rational numbers gives a numbered basis for the topology $\tau$, which makes $(\mathrm{CL}([0,1]), \tau)$ an effective topological space.

Intuitively, describing a closed set $C$ in the topology $\tau$ amounts to giving approximations of $C$ in the hit-or-miss topology and at the same time enumerating the interior of $C$, which is equivalent to giving approximations of both $C$ and $(\operatorname{int}(C))^{c}$ in the hit-or-miss topology.

Proposition 4.5.1. The space $(C L([0,1]), \tau)$ is an effective Polish space.

Proof. The space can be embedded into $\mathrm{CL}([0,1]) \times \mathrm{CL}([0,1])$ endowed with the product of the hit-or-miss topology, which is an effective Polish space. Indeed, the space is computably homeomorphic to the subset $\left\{\left(C,(\operatorname{int}(C))^{c}\right): C \in \mathrm{CL}([0,1])\right\}$ of $\mathrm{CL}([0,1]) \times \mathrm{CL}([0,1])$. We show that this subset is a c.e. effective $G_{\delta}$-set, which implies that it is an effective Polish space by Proposition 2.3.3.

Claim 8. The set $\left\{(A, B):(\operatorname{int}(A))^{c} \subseteq B\right\}$ is $\Pi_{1}^{0}$.

Indeed, $(\operatorname{int}(A))^{c} \subseteq B$ is equivalent to $A^{c} \subseteq B$, which holds iff every rational interval $[a, b]$ that intersects $A^{c}$ intersects $B$.

Claim 9. The set $\left\{(A, B): B \subseteq(\operatorname{int}(A))^{c}\right\}$ is $\Pi_{2}^{0}$.

Indeed, $B \subseteq(\operatorname{int}(A))^{c}$ iff every rational interval $(a, b)$ that intersects $B$ also intersects $A^{c}$.

Now it is c.e. The collection of pairs $\left(C,(\operatorname{int}(C))^{c}\right)$ where $C$ ranges over the finite unions of closed rational intervals is dense in it.

The Polish topology $\tau$ induces a notion of 1-genericity that fits with our objectives. 
Proposition 4.5.2. In the space $(C L([0,1]), \tau)$, every 1 -generic element is regular and its boundary contains only weakly 1-generic reals.

Proof. First, the set of regular closed sets is a dense effective $G_{\delta}$-set. Indeed, $C$ is regular iff every rational interval $(a, b)$ is disjoint from $C$ or intersects the interior of $C$. The collection of finite unions of closed rational intervals (regular sets) is dense.

Let $U \subseteq[0,1]$ be a dense effective open set. Let $\mathcal{U} \subseteq \mathrm{CL}([0,1])$ be the collection of closed sets whose boundary is contained in $U$. It is an effective open set in the topology $\tau$ : the boundary of $C$ is contained in $U$ iff $\operatorname{int}(C) \cup C^{c} \cup U$ covers $[0,1]$, which is semi-decidable from a description of $C . \mathcal{U}$ is moreover dense.

Theorem 4.5.1. Let $P$ be a non-empty regular closed set that is $\tau$-generic from inside. The boundary of $P$ contains only weakly-1-generic points.

Proof. Let $U \subseteq[0,1]$ be a dense effective open set. The class $\mathcal{U}$ of regular closed sets whose boundary is contained in $U$ is an effective open class in the topology $\tau$. Now let $P$ be a regular closed set. There exists a sequence $P_{n}$ of finite unions of closed intervals contained in $\operatorname{int}(P)$ and converging to $P$ in the topology $\tau$. As $U$ is dense, the endpoints of the intervals constituting $P_{n}$ can be taken in $U$. Each $P_{n}$ is contained in $P$ (i.e. $P_{n}$ belongs so $S_{P}$ ), and belongs to $\mathcal{U}$, so $\mathcal{U}$ is dense below $P$. If $P$ is $\tau$-generic from inside then $P$ must belong to $\mathcal{U}$, i.e. its boundary must be contained in $U$.

Theorem 4.1.1 directly gives the following result.

Corollary 4.5.1. In the space $(C L([0,1]), \tau)$ there exists a regular $\Pi_{1}^{0}$-class that is generic from inside and whose boundary is perfect.

Proof. Having a perfect boundary is again a dense effective $G_{\delta}$-property in the topology $\tau$.

As a result, the leftmost element of this set is a left-c.e. real that is weakly-1-generic but not generic from the right.

\section{Acknowledgements}

The author wishes to thank Peter Gács, Emmanuel Jeandel and Cristóbal Rojas for discussions on the subject and the anonymous referees for very useful comments that helped improving the readability of the paper. 


\section{References}

[BG11] Vasco Brattka and Guido Gherardi. Weihrauch degrees, omniscience principles and weak computability. J. Symb. Log., 76(1):143-176, 2011.

[Bra01] Vasco Brattka. Computable versions of Baire's category theorem. In Jiri Sgall, Ales Pultr, and Petr Kolman, editors, MFCS, pages 224-235, London, UK, 2001. Springer-Verlag.

[DHL04] Rodney G. Downey, Denis R. Hirschfeldt, and Geoffrey LaForte. Randomness and reducibility. J. Comput. Syst. Sci., 68(1):96-114, 2004.

[DL02] Rodney G. Downey and Geoffrey LaForte. Presentations of computably enumerable reals. Theor. Comput. Sci., 284(2):539-555, 2002.

[Hoy11] Mathieu Hoyrup. Randomness and the ergodic decomposition. In Benedikt Löwe, Dag Normann, Ivan N. Soskov, and Alexandra A. Soskova, editors, CiE, volume 6735 of Lecture Notes in Computer Science, pages 122-131. Springer, 2011.

[Hoy14] Mathieu Hoyrup. Irreversible computable functions. In Ernst W. Mayr and Natacha Portier, editors, 31st International Symposium on Theoretical Aspects of Computer Science (STACS 2014), STACS 2014, March 5-8, 2014, Lyon, France, volume 25 of LIPIcs, pages 362-373. Schloss Dagstuhl - Leibniz-Zentrum fuer Informatik, 2014.

[HY61] J.G. Hocking and G.S. Young. Topology. Addison-Wesley series in mathematics. Dover Publications, 1961.

[Ing81] M.A. Ingrassia. P-genericity for Recursively Enumerable Sets. PhD thesis, University of Illinois at Urbana-Champaign, 1981.

[Mic51] Ernest Michael. Topologies on spaces of subsets. Trans. Amer. Math. Soc., 71:152-182, 1951.

[Mil04] Joseph S. Miller. Degrees of unsolvability of continuous functions. Journal of Symbolic Logic, 69(2):555-584, 2004.

[Nie09] André Nies. Computability and randomness. Oxford logic guides. Oxford University Press, 2009. 
[Par61] K.R. Parthasarathy. On the category of ergodic measures. Ill. J. Math., 5:648-656, 1961.

[Phe01] R. R. Phelps. Lectures on Choquet's Theorem. Springer, Berlin, 2001.

[Sel71] Alan L. Selman. Arithmetical reducibilities I. Mathematical Logic Quarterly, 17(1):335-350, 1971.

[SW05] Frank Stephan and Guohua Wu. Presentations of K-trivial reals and Ksolmogorov complexity. In $C i E$, pages 461-469, Berlin, Heidelberg, 2005. Springer-Verlag.

[V'y97] Vladimir V. V'yugin. Effective convergence in probability and an ergodic theorem for individual random sequences. SIAM Theory of Probability and Its Applications, 42(1):39-50, 1997.

[Wei92] Klaus Weihrauch. The TTE-interpretation of three hierarchies of omniscience principles. Informatik Berichte 130, FernUniversität Hagen, 1992.

[Wei00] Klaus Weihrauch. Computable Analysis. Springer, Berlin, 2000.

[YMT99] Mariko Yasugi, Takakazu Mori, and Yoshiki Tsujii. Effective properties of sets and functions in metric spaces with computability structure. Theoretical Computer Science, 219(1-2):467-486, 1999. 